\title{
Effects of the Non-Homogeneity and Elastic Medium on the Critical Torsional Load of the Orthotropic Cylindrical Shell ${ }^{*}$
}

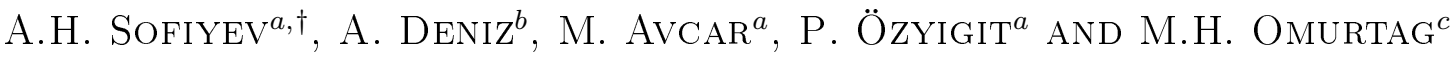 \\ ${ }^{a}$ Department of Civil Engineering, Suleyman Demirel University, Isparta, Turkey \\ ${ }^{b}$ Department of Mathematics, Usak University, Usak, Turkey \\ ${ }^{c}$ Department of Civil Engineering, Istanbul Technical University, Istanbul, Turkey
}

(Received April 26, 2012)

\begin{abstract}
In this study, the torsional stability problem of non-homogeneous orthotropic composite cylindrical shells in the elastic medium, using the Galerkin method was investigated. The Winkler model is used to describe the reaction of the elastic medium on the cylindrical shell. Mixed boundary conditions are considered. The effects of variations of shell parameters, non-homogeneity, orthotropy and foundation stiffness on the critical torsional load are examined.
\end{abstract}

DOI: 10.12693/APhysPolA.123.728

PACS: 61.43.Bn, 62.20.mq

\section{Introduction}

In recent years, non-homogeneous composite shell structures are widely used in modern engineering structures such as tunnels, storage tanks, pressure vessels, water ducts, pipelines, and casing pipes, process equipment and in other applications. Such shells are usually laid on or placed in a soil medium as an elastic foundation, thus there is a great interest in stability analysis of cylindrical shells on the elastic foundation. The non-homogeneity of materials stems from production techniques, surface and thermal polishing processes, effect of radiation, etc. Thus, the mechanical properties of materials change from point to point as continuous functions of coordinates $[1,2]$. Currently, some investigations on the stability analysis of cylindrical shells resting on the elastic foundation have been published in the open literature [3]. There are some important publications related to the torsional stability of composite shells [4]. Hence, the stability of non-homogeneous orthotropic cylindrical shells surrounded by an elastic medium and subjected to torsional load has not been studied enough, analytically. In the current study, an attempt is made to address this problem. The basic equations of non-homogeous orthotropic cylindrical shells resting on the Winkler elastic foundation are derived and closed form solution is obtained. The results reveal that variations of the cylindrical shell parameters, the non-homogeneity, the orthotropy of material and the Winkler foundation stiffness have significant effects on the values of the critical torsional load.

†Paper of the 2nd International Congress on Advances in Applied Physics and Materials Science (APMAS2012), April 26-29, 2012, Antalya, Turkey

${ }^{\dagger}$ corresponding author; e-mail: as of iyev@mmf . sdu.edu.tr

\section{Problem description and basic equations}

As shown in Fig. 1, a thin non-homogeneous orthotropic circular cylindrical shell surrounded by an elastic medium and subjected to the uniform torsional load, $S$, is considered. The cylindrical shell is of length $L$, thickness $h$, and radius $R$. The origin of the coordinate system is taken as the left side of length of the reference surface of the cylindrical shell. The $x$ axis is taken along a generator, $y$ axis is taken tangential directions and $\zeta$ axis normal to them. The axes of orthotropy are parallel to $x$ and $y$ axes. The Winkler model is used to describe the reaction of the elastic medium on the cylindrical shell and $K_{w}\left(\right.$ in $\left.\mathrm{N} / \mathrm{m}^{3}\right)$ is the Winkler foundation stiffness.

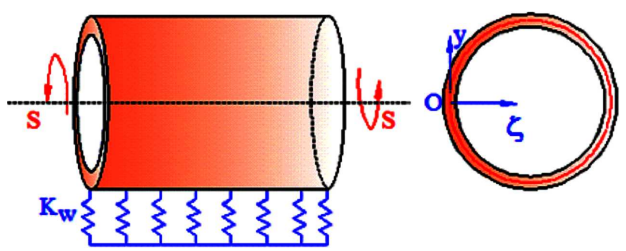

Fig. 1. Geometry of an orthotropic cylindrical shell on the Winkler elastic foundation and under torsion.

The non-homogeneity of ortotropic material of the shell is assumed to arise due to the exponential variation of Young's moduli along the thickness direction $\zeta$ as $[1,2]$ :

$$
\begin{aligned}
& E_{1}(\bar{\zeta})=E_{01} \mathrm{e}^{\mu(\bar{\zeta}-0.5)}, \quad E_{2}(\bar{\zeta})=E_{02} \mathrm{e}^{\mu(\bar{\zeta}-0.5)}, \\
& G(\bar{\zeta})=G_{0} \mathrm{e}^{\mu(\bar{\zeta}-0.5)}, \quad \bar{\zeta}=\zeta / h,
\end{aligned}
$$

where $E_{01}$ and $E_{02}$ are the Young moduli in $x$ and $y$ directions, respectively, $\mathrm{G}_{0}$ is the shear modulus, $\mu$ is the exponential factor characterizing the degree of material gradient in $\zeta$ direction and satisfies $-1 \leq \mu \leq 1$. We remark that $\mu=0$ corresponds to the homogeneous case, $\mu<0$ to the graded soft material, and $\mu>0$ to the graded stiff material. 
TABLE

The variation of critical torsional loads and $\left(n_{\mathrm{cr}}, \gamma_{\mathrm{cr}}\right)$, for $\mathrm{H}$ and $\mathrm{NH}$ orthotropic cylindrical shells on the Winkler elastic foundation versus $R / h, L / R, E_{01} / E_{02}$ and $\mu$ for $K_{w}=5 \times 10^{6} \mathrm{~N} / \mathrm{m}^{3}$.

\begin{tabular}{|c|c|c|c|c|c|c|c|c|}
\hline & \multicolumn{4}{|c|}{$S_{\mathrm{cr}}^{\mathrm{NH}} / 10^{8} \mathrm{~Pa}\left(n_{\mathrm{cr}}, \gamma_{\mathrm{cr}}\right), \mu=0.5$} & \multicolumn{4}{|c|}{$S_{\mathrm{cr}}^{\mathrm{wNH}} / 10^{8} \mathrm{~Pa}\left(n_{\mathrm{cr}}, \gamma_{\mathrm{cr}}\right), \mu=0.5$} \\
\hline$R / h$ & $L / R=0.5$ & $L / R=1$ & $L / R=2$ & $L / R=3$ & $L / R=0.5$ & $L / R=1$ & $L / R=2$ & $L / R=3$ \\
\hline 75 & $2.222(0.32,12)$ & $0.784(0.33,8)$ & $0.423(0.31,7)$ & $0.346(0.27,6)$ & $2.252(0.32,12)$ & $0.839(0.34,9)$ & $0.508(0.36,8)$ & $0.441(0.35,7)$ \\
\hline 100 & $1.299(0.32,13)$ & $0.499(0.33,9)$ & $0.293(0.30,8)$ & $0.244(0.26,7)$ & $1.345(0.33,13)$ & $0.577(0.36,10)$ & $0.395(0.38,9)$ & $0.362(0.38,9)$ \\
\hline 125 & $0.864(0.32,13)$ & $0.356(0.32,10)$ & $0.222(0.28,8)$ & $0.189(0.27,8)$ & $0.930(0.34,14)$ & $0.454(0.38,11)$ & $0.342(0.41,10)$ & $0.321(0.41,10)$ \\
\hline$R / h$ & \multicolumn{4}{|c|}{$S_{\mathrm{cr}}^{\mathrm{H}} / 10^{8} \mathrm{~Pa}\left(n_{\mathrm{cr}}, \gamma_{\mathrm{cr}}\right), \mu=0$} & \multicolumn{4}{|c|}{$S_{\mathrm{cr}}^{\mathrm{wH}} / 10^{8} \mathrm{~Pa}\left(n_{\mathrm{cr}}, \gamma_{\mathrm{cr}}\right), \mu=0$} \\
\hline 75 & $2.857(0.32,12)$ & $1.006(0.33,8)$ & $0.541(0.31,7)$ & $0.442(0.27,6)$ & $2.887(0.32,12)$ & $1.063(0.34,9)$ & $0.629(0.35,7)$ & $0.542(0.34,7)$ \\
\hline 100 & $1.671(0.32,13)$ & $0.640(0.33,9)$ & $0.375(0.30,8)$ & $0.313(0.26,7)$ & $1.717(0.33,13)$ & $0.721(0.35,10)$ & $0.484(0.37,9)$ & $0.441(0.37,9)$ \\
\hline \multirow[t]{2}{*}{125} & $1.111(0.32,13)$ & $0.457(0.32,10)$ & $0.284(0.28,8)$ & $0.242(0.27,8)$ & $1.178(0.33,14)$ & $0.558(0.37,11)$ & $0.411(0.39,10)$ & $0.384(0.40,10)$ \\
\hline & \multicolumn{7}{|c|}{$S_{\mathrm{cr}} / 10^{8} \mathrm{~Pa}\left(n_{\mathrm{cr}}, \gamma_{\mathrm{cr}}\right), R / h=100, L / R=2$} & \\
\hline$E_{01} / E_{02}$ & $\mathrm{NH}(\mu=0.5)$ & $\mathrm{H}$ & $\mathrm{wNH}(\mu=0.5)$ & $\mathrm{wH}$ & $\mu$ & NH & $\mathrm{wNH}$ & \\
\hline 5 & $1.031(0.22,8)$ & $1.321(0.22,8)$ & $1.183(0.25,9)$ & $1.483(0.25,9)$ & -1.0 & $0.624(0.30,8)$ & $0.744(0.34,8)$ & \\
\hline 25 & $0.426(0.18,9)$ & $0.545(0.18,9)$ & $0.549(0.23,10)$ & $0.672(0.22,10)$ & 0 & $0.375(0.30,8)$ & $0.484(0.37,9)$ & \\
\hline 50 & $0.292(0.17,10)$ & $0.374(0.17,10)$ & $0.406(0.22,11)$ & $0.493(0.21,11)$ & 1.0 & $0.229(0.30,8)$ & $0.327(0.39,9)$ & \\
\hline
\end{tabular}

Stress-strain relations for thin non-homogeneous orthotropic cylindrical shells are

$$
\left(\begin{array}{c}
\sigma_{11} \\
\sigma_{12} \\
\sigma_{12}
\end{array}\right)=\left[\begin{array}{ccc}
Q_{11} & Q_{12} & 0 \\
Q_{21} & Q_{22} & 0 \\
0 & 0 & Q_{66}
\end{array}\right]\left[\begin{array}{l}
e_{11}-\zeta w_{, x x} \\
e_{12}-\zeta w_{, y y} \\
e_{12}-2 \zeta w_{, x y}
\end{array}\right],
$$

where $\sigma_{i j}(i, j=1,2)$ are the stress components, $e_{i j}(i, j=1,2)$ are the strain components on the reference surface, $w$ is the displacement of the reference surface in the normal direction and assumed to be much smaller than the thickness, and coma indicates the partial derivative. The quantities $Q_{i j}, i, j=1,2,6$ are given in Refs. [1, 2].

The basic equations of non-homogeneous orthotropic cylindrical shells under torsional load and resting on the Winkler elastic foundation can be obtained as

$$
\begin{aligned}
& c_{12} \Psi_{, x x x x}+\left(c_{11}-2 c_{31}+c_{22}\right) \Psi_{, x x y y}+c_{21} \Psi_{, y y y y} \\
& \quad+\frac{1}{R} \Psi_{, x x}-c_{13} w_{, x x x x}-\left(c_{14}+2 c_{32}+c_{23}\right) w_{, x x y y} \\
& \quad-c_{24} w_{, y y y y}-2 S h w_{, x y}+K_{w} w=0, \\
& b_{22} \Psi_{, x x x x}+\left(b_{12}+2 b_{31}+b_{21}\right) \Psi_{, x x y y}+b_{11} \Psi_{, y y y y} \\
& \quad-b_{23} w_{, x x x x}-\left(b_{13}-2 b_{32}+b_{24}\right) w_{, x x y y} \\
& \quad-b_{14} w_{, y y y y}+\frac{1}{R} w_{, x x}=0,
\end{aligned}
$$

where $c_{i j}, b_{j}(j=1,2 \ldots, 4)$ are non-homogeneous orthotropic shell characteristics [2].

\section{Solution of the problem}

The edge conditions of the cylindrical shell are mixed boundary conditions. The solution of Eqs. (3) and (4) is sought in the following form [5]:

$$
\begin{aligned}
& w=\xi_{1} \sin \frac{\pi x}{L} \sin \frac{n}{R}(y+\gamma x), \\
& \Psi=\xi_{2} \sin \frac{\pi x}{L} \sin \frac{n}{R}(y+\gamma x),
\end{aligned}
$$

where $m_{1}=\pi R / L, n$ is the wave number in the direction of $y$ axis, $\gamma$ is tangent of the angle between the waves and $x$ axis, $\xi_{1}$ and $\xi_{2}$ are amplitudes $[4,5]$.
Substituting Eq. (5) into Eqs. (3) and (4), and then applying the Galerkin method, the expression for the critical torsional load (in $\mathrm{MPa}$ ) of non-homogeneous orthotropic cylindrical shells resting on the Winkler elastic foundation is obtained

$$
\begin{aligned}
& S_{\mathrm{cr}}^{\mathrm{wNH}}=\frac{1}{2 h n^{2} \gamma R^{2}}\left\{\left[R\left(\gamma^{2} n^{2}+m_{1}^{2}\right)\right.\right. \\
& \quad-c_{12}\left(m_{1}^{4}+\left(c_{21} / c_{12}\right) n^{4}+6 m_{1}^{2} n^{2} \gamma^{2}+n^{4} \gamma^{4}\right) \\
& \left.\quad-\left(c_{11}-2 c_{31}+c_{22}\right)\left(n^{4} \gamma^{2}+m_{1}^{2} n^{2}\right)\right] \\
& \quad \times\left[b_{23}\left(m_{1}^{4}+\left(b_{14} / b_{23}\right) n^{4}+6 m_{1}^{2} n^{2} \gamma^{2}+\gamma^{4} n^{4}\right)\right. \\
& +\left(b_{13}-2 b_{32}+b_{24}\right)\left(n^{4} \gamma^{2}+m_{1}^{2} n^{2}\right) \\
& \left.+\left(\gamma^{2} n^{2}+m_{1}^{2}\right) R\right] \\
& \quad /\left[b_{22}\left(m_{1}^{4}+\left(b_{11} / b_{22}\right) n^{4}+6 m_{1}^{2} n^{2} \gamma^{2}+\gamma^{4} n^{4}\right)\right. \\
& \left.+\left(b_{12}+2 b_{31}+b_{21}\right)\left(n^{4} \gamma^{2}+m_{1}^{2} n^{2}\right)\right] \\
& +c_{13}\left(6 m_{1}^{2} n^{2} \gamma^{2}+m_{1}^{4}+\left(c_{24} / c_{13}\right) n^{4}+\gamma^{4} n^{4}\right) \\
& \left.+\left(c_{14}+2 c_{32}+c_{23}\right)\left(n^{4} \gamma^{2}+m_{1}^{2} n^{2}\right)+K_{w} R^{4}\right\}
\end{aligned}
$$

The minimum values of the critical torsional load are obtained by minimizing Eq. (6) with respect to $(n, \gamma)$.

\section{Numerical examples and discussions}

Numerical examples, for the homogeneous $(\mathrm{H})$ and non-homogeneous (NH) orthotropic cylindrical shells with or without Winkler elastic foundation are presented in Table. The calculations were performed for the ratio $E_{01} / E_{02}$, and $E_{01}=2 \times 10^{11} \mathrm{~Pa}, \nu_{12}=0.3$ are taken into account [2]. In all the other computations, boron-epoxy composite with the following orthotropic material properties are considered: $E_{01}=2.06844 \times 10^{11} \mathrm{~Pa}, E_{02}=$ $1.86159 \times 10^{10} \mathrm{~Pa}, G_{0}=4.48162 \times 10^{9} \mathrm{~Pa}, \nu_{12}=0.21$, $\nu_{21}=0.0189$ [6]. As the ratios $R / h, L / R, E_{01} / E_{02}$ and exponential factor $\mu$ increase, the values of the critical 
torsional load and the effect of non-homogeneity decrease for the $\mathrm{H}$ and $\mathrm{NH}$ orthotropic cylindrical shells with or without an elastic foundation. The effect of the elastic foundation on the critical torsional loads increases, as $R / h, L / R, E_{01} / E_{02}$ and $\mu$ increase. It is seen that $S_{\mathrm{cr}}^{\mathrm{NH}}>S_{\mathrm{cr}}^{\mathrm{H}}$ for the soft material, whereas, $S_{\mathrm{cr}}^{\mathrm{NH}}<S_{\mathrm{cr}}^{\mathrm{H}}$ for the stiff material.

\section{Conclusions}

In this study, the torsional stability problem of non-homogeneous orthotropic composite cylindrical shells on the elastic foundation, using the Galerkin method was investigated. The Winkler model is used to describe the reaction of the elastic medium on the cylindrical shell. Mixed boundary conditions are considered. The effects of variations of shell parameters, non-homogeneity, orthotropy and foundation stiffness on the critical torsional load are examined.

\section{Acknowledgments}

The authors thank TUBITAK for the support of the project number 110M695.

\section{References}

[1] E. Pan, J. Compos. Mater. 37, 1913 (2003).

[2] A.H. Sofiyev, M. Omurtag, E. Schnack, J. Sound Vib. 319, 963 (2009).

[3] S.L. Fok, J. Strain Anal. Eng. Des. 37, 375 (2002).

[4] X.Q. Zhang, Q. Han, Thin-Walled Struct. 45, 1035 (2007).

[5] A.S. Volmir, The Stability of Deformable Systems, Nauka, Moscow 1967 (in Russian).

[6] J.N. Reddy, Mechanics of Laminated Composite Plates and Shells, 2nd ed., CRC Press, New York 2004. 